\title{
Longitudinal sleep duration and subsequent development of cancer in the Japanese population
}

\author{
D. KOBAYASHI ${ }^{1,2,3, *}$, T. SHIMBO ${ }^{4}$ \\ ${ }^{1}$ Division of General Internal Medicine, Department of Medicine, St. Luke's International Hospital, Tokyo, Japan; ${ }^{2}$ Department of Epidemiology, \\ St. Luke's International University Graduate School of Public Health, Tokyo, Japan; ${ }^{3}$ Fujita Health University, Toyoake, Japan, ${ }^{4}$ Ohta Nishinouchi \\ Hospital, Koriyama, Japan
}

${ }^{*}$ Correspondence: daikoba@luke.ac.jp

Received February 19, 2020 / Accepted May 26, 2020

\begin{abstract}
Evidence of the association between sleep duration and subsequent cancer development is inconsistent. The aim of this study was to evaluate the association between sleep duration and any type of cancer incidences in the Japanese population. A retrospective longitudinal study was conducted from 2005 to 2018 at St. Luke's International Hospital, Tokyo, Japan. All participants were $\geq 50$ years old and underwent health check-ups at the Center for Preventive medicine at the hospital. Our outcome was the development of any type of cancer during follow-up. The outcomes were evaluated across the sleep duration categories with a longitudinal analysis adjusted for potential confounders. A total of 24,873 participants were included in this study; the mean age was 59.1 years (standard deviation: 7.2) and 13,258 (53.3\%) were male. During the median follow-up of 1,841 days (interquartile range: 821-3,246); 2,544 (10.2\%) developed a type of cancer. After adjusting for potential confounders, the development of any type of cancer did not vary across the sleep duration categories compared to the reference group (6-7 hours) (adjusted ORs were from 0.95 to 1.15, all were statistically insignificant). In terms of each type of cancer, sleep duration was associated with malignant neoplasms of digestive organs associated with a $U$ shape and long sleep duration was associated with a higher incidence of malignant neoplasms of lymphoid, hematopoietic, and related tissues (OR 15.9, 95\%CI: 1.61-156) among females. According to our longitudinal study, there was no clear association between sleep duration and subsequent development of any type of cancer. However, sleep duration was associated with certain types of cancer such as that of digestive organs, lymphoid, hematopoietic, and related tissues in females, and genital organs in males.
\end{abstract}

Key words: short sleep duration, cancer, longitudinal study, ICD-10

The possible association between sleep duration and subsequent cancer was suspected and examined by many researchers, but none have reached a clear conclusion. Many cohort studies and some meta-analyses failed to develop consistent evidence of the association [1-3]. Some studies suggested that short sleep duration was associated with a higher risk of cancer $[4,5]$, while others suggest a long sleep duration [6, 7]. However, combined findings showed no association between sleep duration and subsequent cancer incidence [1]. Therefore, the evidence is unclear; the true association between sleep duration and cancer development still requires investigation.

Evidence about the association is even more complicated and limited when we consider types of cancer or race [1]. Most previous studies focused on specific cancers, such as breast cancer, and studies about other types of cancer are limited $[1,3]$. Unreported studies evaluated certain types of cancer development and failed to show meaningful associations to sleep duration. This may be caused by publication bias and affected the pooled research. In addition, racial differences have been reported for the association between sleep duration and subsequent cancer [1]. Therefore, studies that evaluate the association with each type of cancer incidence for a specific population would be required to add more evidence on this topic.

In addition, a longitudinal study would give us additional insight into the topic. Almost all previous studies evaluated the associations with baseline sleep duration. However, as we reported in the previous study [8], several people changed their sleep duration in their lifetime. In addition, other cancer-related health habits or comorbidities also change during their lifetime. Therefore, a longitudinal study would be useful to evaluate a more precise association between sleep duration and subsequent development of cancer. 
The aim of this study was to evaluate the association between sleep duration and any/each type of cancer incidence in the Japanese population through a longitudinal study design.

\section{Patients and methods}

We conducted a longitudinal study to evaluate the association between sleep duration and subsequent development of cancer from 2005 to 2018 at St. Luke's International Hospital, Tokyo Japan. St. Luke's International Hospital Ethics Committee approved this study (approval number: 18-R203; Comprehensive approvals for studies about social habits using data from health check-ups at center for preventive medicine at the hospital). We included all participants who were $\geq 50$ years old and underwent health check-ups at the Center for Preventive Medicine at the hospital. We excluded participants with a history of any type of cancer. Some participants opted-out of the study. Our outcome was the development of any type of cancer during follow up. The outcomes were evaluated across sleep duration with longitudinal analyses adjusted for potential confounders.

Outcomes. Our primary outcome was the development of any type of cancer during follow-up. The cancer development information was obtained based on the medical records in the hospital, participants' self-report as a part of a questionnaire during the health check-up, and referral of documents from other healthcare institutes. As secondary outcomes, we evaluated each type of cancer, based on the international classification of disease 10 (ICD-10) [9]. All the information was followed up to December 31, 2018. If the participant developed cancers multiple times, we used only the data of the first episode of cancer development for analyses.

Sleep duration. As part of the health check-ups, participants answered questions about their sleep duration on a typical day. We categorized the sleep duration into 5 groups: $\leq 5$ hours; $5-6$ hours; $6-7$ hours; $7-8$ hours; and $\geq 8$ hours, defining sleep duration of 6-7 hours as the reference group based on the previous study [10]. The information about sleep duration was obtained at each visit and dealt with as a time-dependent variable. We considered the reverse causation, where cancer may cause sleep disturbance by either shortening or prolonging sleep duration. We excluded sleep duration information from the previous year, for participants diagnosed with any type of cancer to exclude the effects of the reverse causation.

Adjustment variables. Adjustment variables included participants' demographics, health habits, body mass index (BMI), comorbidities (diabetes and hypertension), and family history of any type of cancer. Demographics were the age at the time of visit and gender. Health habits included alcohol consumption (abstainer, occasional drinker, or regular drinker), smoking status (never, former, or current) and exercise habits (almost none, 1-2 times a week, 3-5 times a week, almost every day), comorbidities (hypertension and diabetes), and family history of any type of cancer. These were collected based on self-reports. In terms of BMI, height and weight were measured during the check-ups and categorized into three groups; underweight $\left(<18.5 \mathrm{~kg} / \mathrm{m}^{2}\right)$, normal weight $\left(18.5-24.9 \mathrm{~kg} / \mathrm{m}^{2}\right)$, or overweight/obese $\left(\geq 25.0 \mathrm{~kg} / \mathrm{m}^{2}\right)$ based on the Asian criteria [11]. All the information was collected at each visit and dealt with time-dependent variables.

Statistical analysis. First, we compared participants' baseline characteristics to the sleep duration category. Next, we compared outcomes to the category with longitudinal analysis. The time-varying Cox proportional hazard model was applied to calculate the adjusted hazard ratio (HR) of the development of cancer by the sleep duration category. We adjusted for the adjustment variables described above. As a sub-analysis, we stratified participants by gender and baseline age (50-64 years, $\geq 65$ years) to examine whether the effects of sleep duration on developing cancer were different. All analyses were performed in 2019 using Stata 14.0 (StataCorp, TX, USA).

\section{Results}

Baseline participant's characteristics. A total of 24,873 participants were included in this study; the mean age was 59.1 (standard deviation: 7.2 ) years and 13,258 (53.3\%) were males. Table 1 and Table 2 show the participants' baseline characteristics by the sleep duration category. Shorter sleepers tended to be younger and mostly female compared to longer sleepers. The health habits analysis for short sleepers revealed that they consumed less alcohol, most had never smoked, but they did less exercise. The shortest and longest sleepers were mostly obese. There were less hypertension and diabetes reported for shorter sleepers compared to longer sleepers.

Longitudinal association from multivariate analyses. During median follow-up of 1,841 (interquartile range: 821-3,246) days; 2,544 (10.2\%) developed some type of cancer. The median interval of each visit was 365 (interquartile range: 357-389) days. After adjusting for potential confounders, the development of any type of cancer did not vary across the sleep duration category (adjusted HRs was from 0.96 to 1.19 , all were not statistically significant, Table 3, Figure 1). In terms of each type of cancer, only C81C96: malignant neoplasms of lymphoid, hematopoietic, and related tissues showed a U-shaped association with statistical significance only in sleep duration of 8 hours or more (HR: $10.1,95 \%$ confidence interval (CI): 1.99-51.4). In the results of subanalysis by gender, the association between sleep duration category and development of any type of cancer mirrored a J-shaped curve only in females (Table 4). Among female participants, a U-shaped association between C15-C26: malignant neoplasms of digestive organs and sleep duration were observed. In addition, long sleep duration (8 hours or more) was associated with higher HR of developing C81C96: malignant neoplasms of lymphoid, hematopoietic, and related tissues. Among male participants, 5-6 hours of sleep 
Table 1. Baseline characteristics and development of cancer by baseline sleep duration category.

\begin{tabular}{|c|c|c|c|c|c|c|c|c|c|}
\hline \multirow[b]{3}{*}{ Age, year (SD) } & \multicolumn{9}{|c|}{ Sleep duration category } \\
\hline & \multicolumn{2}{|c|}{$\begin{array}{l}\leq 5 \text { hours } \\
(n=4,189)\end{array}$} & $\begin{array}{l}5-6 \text { hours } \\
(n=9,847)\end{array}$ & \multicolumn{2}{|c|}{$\begin{array}{l}6-7 \text { hours } \\
(n=7,932)\end{array}$} & \multicolumn{2}{|c|}{$\begin{array}{l}7-8 \text { hours } \\
(n=2,640)\end{array}$} & \multicolumn{2}{|c|}{$\begin{array}{c}\geq 8 \text { hours } \\
(n=265)\end{array}$} \\
\hline & 57.3 & $(6.7)$ & $58.2(6.8)$ & 59.8 & $(7.0)$ & 62.3 & $(7.8)$ & 64.4 & $(8.7)$ \\
\hline Female, n (\%) & 2,193 & $(52.3)$ & $4,710 \quad(47.8)$ & 3,541 & $(44.6)$ & 1,074 & $(40.7)$ & 97 & $(46.6)$ \\
\hline Male, n (\%) & 1,996 & $(47.7)$ & $5,137 \quad(52.2)$ & 4,391 & $(55.4)$ & 1,566 & $(59.3)$ & 168 & $(63.4)$ \\
\hline Development of cancer, n (\%) & 374 & $(8.9)$ & $920 \quad(9.3)$ & 862 & $(10.9)$ & 344 & $(13.0)$ & 44 & $(16.6)$ \\
\hline \multicolumn{10}{|l|}{ Alcohol consumption, $\mathrm{n}(\%)$} \\
\hline Abstainer & 1,743 & $(41.6)$ & $3,987 \quad(40.5)$ & 3,183 & $(40.1)$ & 1,054 & $(39.9)$ & 104 & $(39.3)$ \\
\hline Occasional drinker & 714 & $(7.0)$ & $1,584 \quad(16.1)$ & 1,209 & $(15.2)$ & 345 & $(13.1)$ & 20 & $(7.6)$ \\
\hline Regular drinker & 1,732 & $(41.4)$ & $4,276 \quad(43.4)$ & 3,540 & $(44.6)$ & 1,241 & $(47.0)$ & 141 & $(53.2)$ \\
\hline \multicolumn{10}{|l|}{ Smoking, n (\%) } \\
\hline Never smoker & 2,448 & $(58.4)$ & $5,537 \quad(56.2)$ & 4,352 & $(54.9)$ & 1,359 & $(51.5)$ & 127 & $(47.9)$ \\
\hline Former smoker & 1,118 & $(26.7)$ & $2,888 \quad(29.3)$ & 2,404 & $(30.5)$ & 836 & $(31.7)$ & 101 & $(38.1)$ \\
\hline Current smoker & 623 & $(14.9)$ & $1,422 \quad(14.4)$ & 1,176 & $(14.8)$ & 445 & $(16.9)$ & 37 & $(14.0)$ \\
\hline \multicolumn{10}{|l|}{ Exercise habit, n (\%) } \\
\hline Almost none & 1,508 & $(36.0)$ & $2,852 \quad(29.0)$ & 1,921 & $(24.2)$ & 658 & $(24.9)$ & 80 & $(30.2)$ \\
\hline 1-2 times a week & 1,495 & $(35.7)$ & $3,805 \quad(38.6)$ & 3,085 & $(38.9)$ & 920 & $(34.9)$ & 84 & $(31.7)$ \\
\hline $3-5$ times a week & 643 & $(15.4)$ & $1,906 \quad(19.4)$ & 1,840 & $(23.2)$ & 658 & $(24.9)$ & 54 & $(20.4)$ \\
\hline Almost everyday & 543 & $(13.0)$ & $1,284 \quad(13.0)$ & 1,086 & $(13.7)$ & 404 & $(15.3)$ & 47 & $(17.7)$ \\
\hline \multicolumn{10}{|l|}{ Body mass index, $\mathrm{n}(\%)$} \\
\hline Underweight & 265 & $(6.3)$ & $609 \quad(6.2)$ & 481 & $(6.1)$ & 166 & $(6.3)$ & 21 & $(7.9)$ \\
\hline Normal weight & 2,822 & $(67.4)$ & $6,918 \quad(70.3)$ & 5,813 & $(73.3)$ & 1,878 & $(71.1)$ & 179 & $(67.6)$ \\
\hline Obese/overweight & 1,101 & $(26.3)$ & $2,320 \quad(23.6)$ & 1,638 & $(20.7)$ & 596 & $(22.6)$ & 65 & $(24.5)$ \\
\hline Hypertension, n (\%) & 685 & $(16.4)$ & $1,622 \quad(16.5)$ & 1,439 & $(18.1)$ & 590 & $(22.4)$ & 67 & $(25.3)$ \\
\hline Diabetes, n (\%) & 159 & $(3.8)$ & $412(4.2)$ & 407 & $(5.1)$ & 157 & $(6.0)$ & 24 & $(9.1)$ \\
\hline Family history of cancer, $\mathrm{n}(\%)$ & 1,908 & $(45.6)$ & $4,653 \quad(47.3)$ & 3,742 & $(47.2)$ & 1,156 & $(43.8)$ & 112 & $(42.3)$ \\
\hline
\end{tabular}

duration was associated with lower HRs of development of any type of cancer (HR 0.88, 95\%CI: 0.78-0.999) and C60C63: malignant neoplasms of male genital organs (HR 0.79, 95\%CI: 0.64-0.97) compared to 6-7 hours of sleep duration. C81-C96: malignant neoplasms of lymphoid, hematopoietic, and related tissues showed a U-shaped association even among male participants, but not statistically significant.

When we stratified by the age group, shorter sleep duration tended to be associated with a lower incidence of any types of cancer compared to the reference group among the younger population, especially for 5-6 hours of sleep duration (HR 0.87 , 95\%CI: $0.78-0.98, p=0.02$, Table 5). Similar to subanalysis by gender, 5-6 hours of sleep duration was associated with a lower incidence of C60-C63: malignant neoplasms of male genital organs (HR 0.74, 95\%CI: 0.56-0.97, p=0.03) and more than 8 hours of sleep duration was associated with a higher incidence of with C81-C96: malignant neoplasms of lymphoid, hematopoietic, and related tissues (HR 10.1, 95\%CI: 1.12-91.5, $\mathrm{p}=0.04$ ). In contrast, among the elderly population, sleep duration was not associated with the development of any type of cancer. In terms of each type of cancer, sleep duration showed a U-shaped association to the development of C15-C26: malignant neoplasms of digestive organs with statistical significance in sleep duration of 5-6 hours (HR 1.33, 95\%CI: $1.02-1.75, \mathrm{p}=0.04$ ) and 8 hours or more (HR 1.74, 95\%CI: 1.03-2.96, $\mathrm{p}=0.04$ ).

\section{Discussion}

Our longitudinal study demonstrated that there was no clear association between sleep duration and subsequent development of any type of cancer. However, the association showed a mild $U$ shape, especially in female participants. In terms of each type of cancer, the sleep duration was associated with malignant neoplasms of digestive organs associated with the $U$ shape, and long sleep duration was associated with a higher incidence of malignant neoplasms of lymphoid, hematopoietic, and related tissues among females. In contrast, 5-6 hours of sleep duration was associated with a lower incidence of any type of cancer, including malignant neoplasms of male genital organs, compared to 6-7 hours of sleep duration among males.

We found that the association between sleep duration and subsequent development of any type of cancer in the Japanese 
Table 2. Baseline health habits by baseline sleep duration category, stratified by gender.

\begin{tabular}{|c|c|c|c|c|c|}
\hline \multirow[b]{2}{*}{ Female } & \multicolumn{5}{|c|}{ Sleep duration category } \\
\hline & $\begin{array}{c}\leq 5 \text { hours } \\
(n=2,193)\end{array}$ & $\begin{array}{l}5-6 \text { hours } \\
(n=4,710)\end{array}$ & $\begin{array}{l}6-7 \text { hours } \\
(n=3,541)\end{array}$ & $\begin{array}{l}7-8 \text { hours } \\
(n=1,074)\end{array}$ & $\begin{array}{c}\geq 8 \text { hours } \\
(n=97)\end{array}$ \\
\hline Abstainer & $1,304 \quad(59.5)$ & $2,870 \quad(60.9)$ & $2,210 \quad(62.4)$ & $687 \quad(64.0)$ & $67 \quad(69.1)$ \\
\hline Occasional drinker & $378 \quad(17.2)$ & $766(16.3)$ & $572(16.2)$ & $151 \quad(14.1)$ & $7(7.2)$ \\
\hline Regular drinker & $511 \quad(23.3)$ & $1,074 \quad(22.8)$ & $759 \quad(21.4)$ & $236 \quad(22.0)$ & $23 \quad(23.7)$ \\
\hline \multicolumn{6}{|l|}{ Smoking, n (\%) } \\
\hline Never smoker & $1,802 \quad(82.2)$ & $3,925 \quad(83.3)$ & $3,009 \quad(85.0)$ & $889 \quad(82.8)$ & $83 \quad(85.6)$ \\
\hline Former smoker & $243(11.1)$ & $492 \quad(10.5)$ & $343 \quad(9.7)$ & $110 \quad(10.2)$ & $8 \quad(8.3)$ \\
\hline Current smoker & $148 \quad(6.8)$ & $293(6.2)$ & $189(5.3)$ & $75(7.0)$ & $6 \quad(6.2)$ \\
\hline \multicolumn{6}{|l|}{ Exercise habit, n (\%) } \\
\hline Almost none & $813(37.1)$ & $1,422 \quad(30.2)$ & $874 \quad(24.7)$ & $299(27.8)$ & $34 \quad(35.1)$ \\
\hline 1-2 times a week & $674 \quad(30.7)$ & $1,637 \quad(34.8)$ & $1,307 \quad(36.9)$ & $347 \quad(32.3)$ & $28 \quad(28.9)$ \\
\hline 3-5 times a week & $401 \quad(18.3)$ & $1,053 \quad(22.4)$ & $934 \quad(26.4)$ & $298 \quad(27.8)$ & $22 \quad(22.7)$ \\
\hline Almost everyday & $305 \quad(13.9)$ & $598 \quad(12.7)$ & $426 \quad(12.0)$ & $130(12.1)$ & $13(13.4)$ \\
\hline \multicolumn{6}{|c|}{ Body mass index, n (\%) } \\
\hline Underweight & $240 \quad(11.0)$ & $519(11.0)$ & $395(11.2)$ & $120(11.2)$ & $15(15.5)$ \\
\hline Normal weight & $1,605 \quad(73.2)$ & $3,547 \quad(75.3)$ & $2,766 \quad(78.1)$ & $817 \quad(76.1)$ & $65 \quad(67.0)$ \\
\hline Obese/overweight & $347 \quad(15.8)$ & $644 \quad(13.7)$ & $380 \quad(10.7)$ & $137 \quad(12.8)$ & $17 \quad(17.5)$ \\
\hline Male & $\begin{array}{l}\leq 5 \text { hours } \\
(n=1,996)\end{array}$ & $\begin{array}{l}\text { 5-6 hours } \\
(n=5,137)\end{array}$ & $\begin{array}{l}\text { 6-7 hours } \\
(n=4,391)\end{array}$ & $\begin{array}{l}\text { 7-8 hours } \\
(n=1,566)\end{array}$ & $\begin{array}{c}>8 \text { hours } \\
(n=168)\end{array}$ \\
\hline \multicolumn{6}{|c|}{ Alcohol consumption, n (\%) } \\
\hline Abstainer & $439 \quad(22.0)$ & $1,117 \quad(21.7)$ & $973 \quad(22.2)$ & 367 (23.4) & $37 \quad(22.0)$ \\
\hline Occasional drinker & $336(16.8)$ & $818 \quad(15.9)$ & $637(14.5)$ & $194(12.4)$ & $13(7.7)$ \\
\hline Regular drinker & $1,221 \quad(61.2)$ & $3,202 \quad(62.3)$ & $2,781 \quad(63.3)$ & $1,005 \quad(64.2)$ & $118 \quad(70.2)$ \\
\hline \multicolumn{6}{|l|}{ Smoking, n (\%) } \\
\hline Never smoker & $646 \quad(32.4)$ & $1,612(31.4)$ & $1,343 \quad(30.6)$ & $470 \quad(30.0)$ & $44 \quad(26.2)$ \\
\hline Former smoker & $875 \quad(43.8)$ & $2,396 \quad(46.6)$ & $2,061 \quad(46.9)$ & $726 \quad(46.4)$ & $93 \quad(55.4)$ \\
\hline Current smoker & $475 \quad(23.8)$ & $1,129(22.0)$ & $987 \quad(22.5)$ & $370 \quad(23.6)$ & $31 \quad(18.5)$ \\
\hline \multicolumn{6}{|l|}{ Exercise habit, n (\%) } \\
\hline Almost none & $695 \quad(34.8)$ & $1,430 \quad(27.8)$ & $1,047 \quad(23.8)$ & $359 \quad(22.9)$ & $46 \quad(27.4)$ \\
\hline 1-2 times a week & $821 \quad(41.1)$ & $2,168 \quad(42.2)$ & $1,778 \quad(40.5)$ & $573 \quad(36.6)$ & $56 \quad(33.3)$ \\
\hline 3-5 times a week & $242 \quad(12.1)$ & $853(16.6)$ & $906 \quad(20.6)$ & $360 \quad(23.0)$ & $32(19.1)$ \\
\hline Almost everyday & $238 \quad(11.9)$ & $686(13.4)$ & $660(15.0)$ & $274 \quad(17.5)$ & $34 \quad(20.2)$ \\
\hline \multicolumn{6}{|c|}{ Body mass index, $\mathrm{n}(\%)$} \\
\hline Underweight & $25 \quad(1.3)$ & $90 \quad(1.8)$ & $86(2.0)$ & $46 \quad(2.9$ & $6(3.6)$ \\
\hline Normal weight & $1,217 \quad(61.0)$ & $3,371 \quad(65.6)$ & $3,047 \quad(69.4)$ & $1,061 \quad(67.8)$ & 114 (67.9) \\
\hline Obese/overweight & $754 \quad(37.8)$ & $1,676 \quad(32.6)$ & $1,258 \quad(28.7)$ & $459(29.3)$ & $48 \quad(28.6)$ \\
\hline
\end{tabular}

population is not clear. This is not consistent with the findings of a previous meta-analysis. In the meta-analysis, the short sleep duration was associated with a higher risk of cancer in the Asian population [1]. Another meta-analysis suggested that short sleep duration was associated with a higher incidence of cancer $[3,12]$. We hypothesized the difference in the findings between studies; not only between our study and other studies. Most meta-analyses focused on a specific type of cancer, such as breast cancer or prostate cancer. As a result, pooled estimates were strongly affected by these specific cancers. Unrestricted meta-analysis studies focusing on more than one type of cancer might have different results that are probably closer to our findings.
Our study showed some associations between sleep duration and certain types of cancer, which were partially supported by previous studies. First, both short and long sleep duration was associated with a higher incidence of cancer of digestive organs in females. Two meta-analysis studies reported that long sleep duration was associated with the incidence of colorectal cancer, but not other gastrointestinal cancers $[1,3]$. Another cohort study demonstrated that male short sleepers had a higher risk of gastric cancer [13]. The effect of residual confounders or Helicobacter pylori carcinogenesis through the disrupted immune-inflammation system were said to be the potential underlying mechanisms of the finding. Second, long sleep duration was associated 
Table 3. Adjusted hazard ratios of developing any type and each type of cancer by sleep duration category from time-varying Cox proportional hazard model adjusting for different covariates.

\begin{tabular}{|c|c|c|c|c|c|}
\hline & \multicolumn{5}{|c|}{$\begin{array}{c}\text { Sleep duration category } \\
\text { Adjusted hazard ratio (95\% confidence interval) } \\
\text { p-value }\end{array}$} \\
\hline & $\leq 5$ hours & 5-6 hours & 6-7 hours & 7-8 hours & $\geq 8$ hours \\
\hline Any type of cancer $(\mathrm{n}=2,546)$ & $\begin{array}{l}0.98(0.86-1.10) \\
0.72\end{array}$ & $\begin{array}{l}0.96(0.87-1.05) \\
0.36\end{array}$ & Reference & $\begin{array}{l}1.06(0.94-1.20) \\
0.33\end{array}$ & $\begin{array}{c}1.19(0.90-1.57) \\
0.21\end{array}$ \\
\hline \multicolumn{6}{|l|}{ Each type of cancer } \\
\hline $\begin{array}{l}\text { C00-C14: Malignant neoplasms of the lips, oral } \\
\text { cavity and pharynx }(n=13)\end{array}$ & $\begin{array}{c}0.68(0.14-3.38) \\
0.64\end{array}$ & $\begin{array}{l}0.28(0.06-1.34) \\
0.11\end{array}$ & Reference & $\begin{array}{c}0.28(0.03-2.31) \\
0.24\end{array}$ & - \\
\hline $\begin{array}{l}\text { C15-C26: Malignant neoplasms of digestive } \\
\text { organs }(n=900)\end{array}$ & $\begin{array}{l}0.99(0.80-1.22) \\
0.91\end{array}$ & $\begin{array}{c}1.09(0.92-1.27) \\
0.32\end{array}$ & Reference & $\begin{array}{c}1.06(0.86-1.30) \\
0.60\end{array}$ & $\begin{array}{c}1.49(0.99-2.25) \\
0.06\end{array}$ \\
\hline $\begin{array}{l}\text { C30-C39: Malignant neoplasms of respiratory } \\
\text { and intrathoracic organs }(n=324)\end{array}$ & $\begin{array}{l}0.80(0.55-1.14) \\
0.22\end{array}$ & $\begin{array}{l}0.94(0.72-1.22) \\
0.64\end{array}$ & Reference & $\begin{array}{l}1.05(0.75-1.48) \\
0.76\end{array}$ & $\begin{array}{c}1.32(0.64-2.72) \\
0.45\end{array}$ \\
\hline $\begin{array}{l}\text { C40-C41: Malignant neoplasms of bone and } \\
\text { articular cartilage }(n=1)\end{array}$ & - & - & Reference & - & - \\
\hline $\begin{array}{l}\text { C43-C44: Melanoma and other malignant neo- } \\
\text { plasms of the skin }(n=48)\end{array}$ & $\begin{array}{l}0.74(0.29-1.86) \\
0.52\end{array}$ & $\begin{array}{l}0.77(0.39-1.51) \\
0.45\end{array}$ & Reference & $\begin{array}{l}0.82(0.35-1.96) \\
0.66\end{array}$ & - \\
\hline $\begin{array}{l}\text { C45-C49: Malignant neoplasms of mesothelial } \\
\text { and soft tissue }(n=5)\end{array}$ & - & $\begin{array}{l}1.55(1.16-18.9) \\
0.71\end{array}$ & Reference & - & - \\
\hline $\begin{array}{l}\text { C50-C50: Malignant neoplasms of breast tissue } \\
(\mathrm{n}=279)\end{array}$ & $\begin{array}{l}1.01(0.72-1.41) \\
0.96\end{array}$ & $\begin{array}{l}0.91(0.69-1.21) \\
0.52\end{array}$ & Reference & $\begin{array}{l}0.83(0.53-1.30) \\
0.41\end{array}$ & $\begin{array}{c}0.56(0.13-2.31) \\
0.42\end{array}$ \\
\hline $\begin{array}{l}\text { C51-C58: Malignant neoplasms of female genital } \\
\text { organs }(n=81)\end{array}$ & $\begin{array}{c}1.17(0.62-2.19) \\
0.63\end{array}$ & $\begin{array}{l}0.98(0.57-1.68) \\
0.94\end{array}$ & Reference & $\begin{array}{l}1.22(0.56-2.63) \\
0.62\end{array}$ & $\begin{array}{c}1.00(0.13-7.94) \\
1.00\end{array}$ \\
\hline $\begin{array}{l}\text { C60-C63: Malignant neoplasms of male genital } \\
\text { organs }(n=573)\end{array}$ & $\begin{array}{c}1.09(0.84-1.41) \\
0.53\end{array}$ & $\begin{array}{c}0.79(0.64-0.97) \\
0.03\end{array}$ & Reference & $\begin{array}{l}1.15(0.91-1.44) \\
0.24\end{array}$ & $\begin{array}{c}0.88(0.48-1.62) \\
0.69\end{array}$ \\
\hline $\begin{array}{l}\text { C64-C68: Malignant neoplasms of the urinary } \\
\text { tract }(n=129)\end{array}$ & $\begin{array}{c}1.20(0.69-2.09) \\
0.51\end{array}$ & $\begin{array}{l}1.06(0.68-1.64) \\
0.81\end{array}$ & Reference & $\begin{array}{l}1.18(0.71-1.98) \\
0.52\end{array}$ & $\begin{array}{c}1.51(0.54-4.24) \\
0.44\end{array}$ \\
\hline $\begin{array}{l}\text { C69-C72: Malignant neoplasms of the eyes, brain, } \\
\text { and other parts of central nervous system }(n=6)\end{array}$ & $\begin{array}{l}0.45(0.04-5.28) \\
0.52\end{array}$ & $\begin{array}{l}0.44(0.07-2.85) \\
0.39\end{array}$ & Reference & - & - \\
\hline $\begin{array}{l}\text { C73-C75: Malignant neoplasms of the thyroid } \\
\text { and other endocrine glands }(n=18)\end{array}$ & $\begin{array}{l}0.75(0.19-2.96) \\
0.68\end{array}$ & $\begin{array}{l}0.99(0.36-2.76) \\
0.98\end{array}$ & Reference & - & - \\
\hline $\begin{array}{l}\text { C76-C80: Malignant neoplasms of ill-defined, } \\
\text { other secondary, and unspecified sites }(n=21)\end{array}$ & $\begin{array}{l}0.71(0.19-2.63) \\
0.60\end{array}$ & $\begin{array}{l}0.28(0.07-1.04) \\
0.06\end{array}$ & Reference & $\begin{array}{c}1.06(0.36-3.19) \\
0.91\end{array}$ & - \\
\hline $\begin{array}{l}\text { C81-C96: Malignant neoplasms of lymphoid, } \\
\text { hematopoietic, and related tissues }(n=29)\end{array}$ & $\begin{array}{c}1.35(0.38-4.84) \\
0.64\end{array}$ & $\begin{array}{l}1.88(0.71-4.97) \\
0.20\end{array}$ & Reference & $\begin{array}{l}1.92(0.54-6.87) \\
0.31\end{array}$ & $\begin{array}{c}10.1(1.99-51.4) \\
<0.01\end{array}$ \\
\hline D00-D09: In situ neoplasms ( $\mathrm{n}=2)$ & - & - & Reference & - & - \\
\hline Unknown $(\mathrm{n}=117)$ & $\begin{array}{c}0.83(0.46-1.49) \\
0.54 \\
\end{array}$ & $\begin{array}{c}1.07(0.70-1.64) \\
0.75 \\
\end{array}$ & Reference & $\begin{array}{c}0.90(0.49-1.67) \\
0.74 \\
\end{array}$ & $\begin{array}{c}0.55(0.08-4.03) \\
0.56 \\
\end{array}$ \\
\hline
\end{tabular}

Models were adjusted for time variable, participants' age and gender, social history (smoking, alcohol consumption, and exercise), body mass index, comorbidities (hypertension, diabetes, and dyslipidemia) and family history of any cancer. Number in bold represents $\mathrm{p}<0.05$.

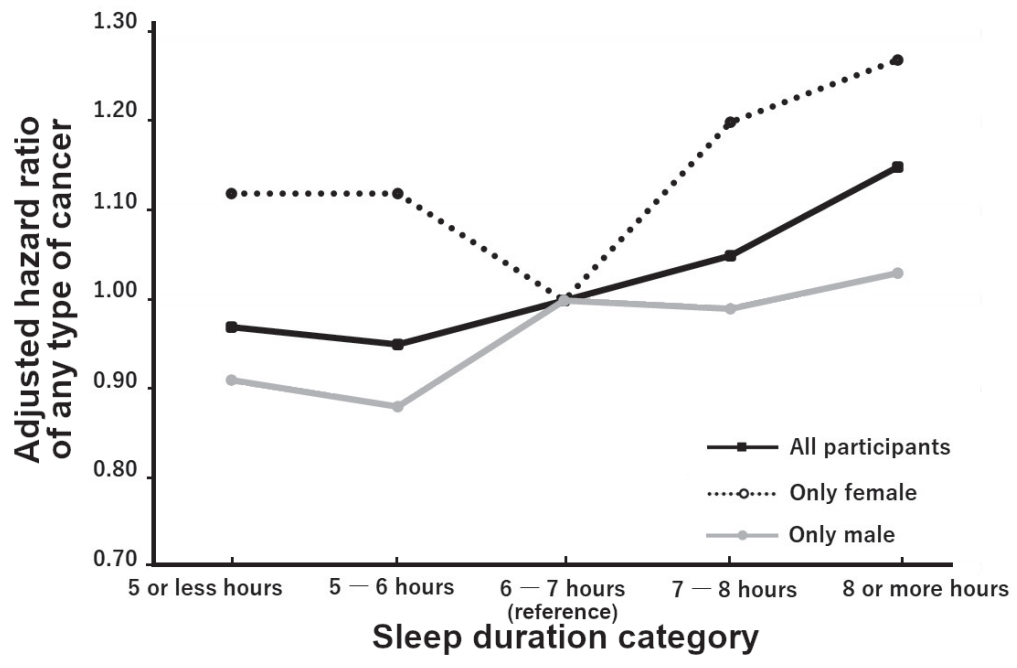

Figure 1. The adjusted hazard ratio of any type of cancer by sleep duration category. Although the adjusted hazard ratios of any type of cancer did not vary across the sleep duration category among all participants or only males, those showed a $U$-shaped association among females. 
Table 4. Adjusted hazard ratios of developing any type and each type of cancer by sleep duration category stratified by gender from time-varying Cox proportional hazard model adjusting for different covariates.

\begin{tabular}{|c|c|c|c|c|c|}
\hline & \multicolumn{5}{|c|}{$\begin{array}{c}\text { Sleep duration category } \\
\text { Adjusted hazard ratio (95\% confidence interval) } \\
\text { p-value }\end{array}$} \\
\hline & $\leq 5$ hours & 5-6 hours & 6-7 hours & $7-8$ hours & $\geq 8$ hours \\
\hline \multicolumn{6}{|l|}{ Females $(n=11,615)$} \\
\hline Any type of cancer $(n=956)$ & $\begin{array}{l}1.12(0.93-1.35) \\
0.25\end{array}$ & $\begin{array}{l}1.12(0.95-1.31) \\
0.17\end{array}$ & Reference & $\begin{array}{l}1.20(0.96-1.50) \\
0.10\end{array}$ & $\begin{array}{l}1.27(0.76-2.14) \\
0.37\end{array}$ \\
\hline $\begin{array}{l}\text { C00-C14: Malignant neoplasms of the lips, oral } \\
\text { cavity and pharynx }(n=5)\end{array}$ & $\begin{array}{l}1.04(0.08-13.0) \\
0.98\end{array}$ & $\begin{array}{l}0.41(0.03-4.95) \\
0.49\end{array}$ & Reference & $\begin{array}{l}1.34(0.10-18.1) \\
0.82\end{array}$ & - \\
\hline $\begin{array}{l}\text { C15-C26: Malignant neoplasms of digestive } \\
\text { organs }(n=301)\end{array}$ & $\begin{array}{l}1.32(0.93-1.86) \\
0.12\end{array}$ & $\begin{array}{l}1.40(1.05-1.87) \\
0.02\end{array}$ & Reference & $\begin{array}{l}1.26(0.85-1.88) \\
0.25\end{array}$ & $\begin{array}{c}2.00(0.90-4.42) \\
0.09\end{array}$ \\
\hline $\begin{array}{l}\text { C30-C39: Malignant neoplasms of respiratory } \\
\text { and intrathoracic organs }(n=145)\end{array}$ & $\begin{array}{l}0.97(0.60-1.57) \\
0.90\end{array}$ & $\begin{array}{l}0.75(0.49-1.13) \\
0.17\end{array}$ & Reference & $\begin{array}{l}1.46(0.89-2.40) \\
0.13\end{array}$ & $\begin{array}{l}1.52(0.51-4.54) \\
0.45\end{array}$ \\
\hline $\begin{array}{l}\text { C40-C41: Malignant neoplasms of bone and } \\
\text { articular cartilage }(n=1)\end{array}$ & - & - & Reference & - & - \\
\hline $\begin{array}{l}\text { C43-C44: Melanoma and other malignant neo- } \\
\text { plasms of the skin }(n=18)\end{array}$ & $\begin{array}{l}0.47(0.10-2.29) \\
0.35\end{array}$ & $\begin{array}{l}0.87(0.30-2.50) \\
0.80\end{array}$ & Reference & $\begin{array}{l}0.74(0.15-3.64) \\
0.71\end{array}$ & - \\
\hline $\begin{array}{l}\text { C45-C49: Malignant neoplasms of mesothelial } \\
\text { and soft tissue }(n=4)\end{array}$ & - & - & Reference & - & - \\
\hline $\begin{array}{l}\text { C50-C50: Malignant neoplasms of breast tissue } \\
(\mathrm{n}=278)\end{array}$ & $\begin{array}{l}1.02(0.73-1.43) \\
0.92\end{array}$ & $\begin{array}{l}0.92(0.69-1.22) \\
0.57\end{array}$ & Reference & $\begin{array}{l}0.83(0.53-1.31) \\
0.43\end{array}$ & $\begin{array}{c}0.56(0.13-2.33) \\
0.43\end{array}$ \\
\hline $\begin{array}{l}\text { C51-C58: Malignant neoplasms of female genital } \\
\text { organs }(n=81)\end{array}$ & $\begin{array}{l}1.17(0.62-2.19) \\
0.63\end{array}$ & $\begin{array}{l}0.98(0.57-1.68) \\
0.94\end{array}$ & Reference & $\begin{array}{l}1.22(0.56-2.63) \\
0.62\end{array}$ & $\begin{array}{c}1.00(0.13-7.94) \\
1.00\end{array}$ \\
\hline $\begin{array}{l}\text { C60-C63: Malignant neoplasms of male genital } \\
\text { organs }(n=0)\end{array}$ & - & - & Reference & - & - \\
\hline $\begin{array}{l}\text { C64-C68: Malignant neoplasms of the urinary } \\
\text { tract }(n=35)\end{array}$ & $\begin{array}{l}2.13(0.71-6.41) \\
0.18\end{array}$ & $\begin{array}{l}2.22(0.86-5.75) \\
0.10\end{array}$ & Reference & $\begin{array}{l}2.32(0.70-7.65) \\
0.17\end{array}$ & $\begin{array}{c}3.37(0.44-30.9) \\
0.23\end{array}$ \\
\hline $\begin{array}{l}\text { C69-C72: Malignant neoplasms of the eyes, brain, } \\
\text { and other parts of central nervous system }(n=5)\end{array}$ & $\begin{array}{l}0.49(0.03-7.38) \\
0.60\end{array}$ & $\begin{array}{l}0.52(0.06-4.19) \\
0.54\end{array}$ & Reference & - & - \\
\hline $\begin{array}{l}\text { C73-C75: Malignant neoplasms of the thyroid } \\
\text { and other endocrine glands }(n=17)\end{array}$ & $\begin{array}{l}0.83(0.20-3.35) \\
0.79\end{array}$ & $\begin{array}{l}1.14(0.39-3.32) \\
0.81\end{array}$ & Reference & - & - \\
\hline $\begin{array}{l}\text { C76-C80: Malignant neoplasms of ill-defined, } \\
\text { other secondary, and unspecified sites }(n=8)\end{array}$ & $\begin{array}{l}1.13(0.18-7.12) \\
0.90\end{array}$ & $\begin{array}{l}0.55(0.09-3.49) \\
0.53\end{array}$ & Reference & $\begin{array}{l}0.97(0.10-9.67) \\
0.98\end{array}$ & - \\
\hline $\begin{array}{l}\text { C81-C96: Malignant neoplasms of lymphoid, } \\
\text { hematopoietic, and related tissues }(n=14)\end{array}$ & $\begin{array}{l}0.61(0.06-5.86) \\
0.67\end{array}$ & $\begin{array}{l}1.94(0.50-7.53) \\
0.34\end{array}$ & Reference & $\begin{array}{l}2.31(0.38-13.9) \\
0.36\end{array}$ & $\begin{array}{l}15.7(1.59-156) \\
0.02\end{array}$ \\
\hline D00-D09: In situ neoplasms $(\mathrm{n}=0)$ & - & - & Reference & - & - \\
\hline Unknown $(\mathrm{n}=46)$ & $\begin{array}{l}1.21(0.42-3.51) \\
0.73\end{array}$ & $\begin{array}{l}2.44(1.10-5.44) \\
0.03\end{array}$ & Reference & $\begin{array}{l}2.74(0.99-7.60) \\
0.05\end{array}$ & - \\
\hline \multicolumn{6}{|l|}{ Males $(n=13,258)$} \\
\hline Any type of cancer $(n=1,588)$ & $\begin{array}{l}0.91(0.77-1.07) \\
0.27\end{array}$ & $\begin{array}{l}0.88(0.78-0.99) \\
0.03\end{array}$ & Reference & $\begin{array}{l}0.99(0.85-1.14) \\
0.87\end{array}$ & $\begin{array}{c}1.03(0.74-1.43) \\
0.86\end{array}$ \\
\hline $\begin{array}{l}\text { C00-C14: Malignant neoplasms of the lips, oral } \\
\text { cavity and pharynx }(n=8)\end{array}$ & $\begin{array}{l}0.64(0.07-5.64) \\
0.69\end{array}$ & $\begin{array}{l}0.20(0.02-1.68) \\
0.14\end{array}$ & Reference & - & - \\
\hline $\begin{array}{l}\text { C15-C26: Malignant neoplasms of digestive } \\
\text { organs }(n=599)\end{array}$ & $\begin{array}{l}0.84(0.63-1.10) \\
0.20\end{array}$ & $\begin{array}{l}0.96(0.79-1.16) \\
0.65\end{array}$ & Reference & $\begin{array}{l}0.98(0.77-1.25) \\
0.90\end{array}$ & $\begin{array}{c}1.33(0.82-2.17) \\
0.25\end{array}$ \\
\hline $\begin{array}{l}\text { C30-C39: Malignant neoplasms of respiratory } \\
\text { and intrathoracic organs }(n=179)\end{array}$ & $\begin{array}{l}0.63(0.36-1.12) \\
0.12\end{array}$ & $\begin{array}{l}1.13(0.80-1.59) \\
0.49\end{array}$ & Reference & $\begin{array}{l}0.82(0.52-1.31) \\
0.41\end{array}$ & $\begin{array}{l}0.88(0.32-2.44) \\
0.81\end{array}$ \\
\hline $\begin{array}{l}\text { C40-C41: Malignant neoplasms of bone and } \\
\text { articular cartilage }(n=0)\end{array}$ & - & - & Reference & - & - \\
\hline $\begin{array}{l}\text { C43-C44: Melanoma and other malignant neo- } \\
\text { plasms of the skin }(n=30)\end{array}$ & $\begin{array}{l}0.93(0.30-2.90) \\
0.90\end{array}$ & $\begin{array}{l}0.67(0.28-1.64) \\
0.39\end{array}$ & Reference & $\begin{array}{l}0.85(0.30-2.40) \\
0.76\end{array}$ & - \\
\hline $\begin{array}{l}\text { C45-C49: Malignant neoplasms of mesothelial } \\
\text { and soft tissue }(n=1)\end{array}$ & - & - & Reference & - & - \\
\hline $\begin{array}{l}\text { C50-C50: Malignant neoplasms of breast tissue } \\
(\mathrm{n}=1)\end{array}$ & - & - & Reference & - & - \\
\hline $\begin{array}{l}\text { C51-C58: Malignant neoplasms of female genital } \\
\text { organs }(n=0)\end{array}$ & - & - & Reference & - & - \\
\hline
\end{tabular}




\begin{tabular}{|c|c|c|c|c|c|}
\hline & \multicolumn{5}{|c|}{$\begin{array}{l}\text { Sleep duration category } \\
\text { Adjusted hazard ratio (95\% confidence interval) } \\
\text { p-value }\end{array}$} \\
\hline & $\leq 5$ hours & 5-6 hours & 6-7 hours & 7-8 hours & $\geq 8$ hours \\
\hline $\begin{array}{l}\text { C60-C63: Malignant neoplasms of male genital } \\
\text { organs }(n=573)\end{array}$ & $\begin{array}{c}1.09(0.84-1.41) \\
0.53\end{array}$ & $\begin{array}{l}0.79(0.64-0.97) \\
0.03\end{array}$ & Reference & $\begin{array}{l}1.15(0.91-1.44) \\
0.24\end{array}$ & $\begin{array}{l}0.88(0.48-1.62) \\
0.69\end{array}$ \\
\hline $\begin{array}{l}\text { C64-C68: Malignant neoplasms of the urinary } \\
\text { tract }(n=94)\end{array}$ & $\begin{array}{l}1.01(0.52-1.97) \\
0.97\end{array}$ & $\begin{array}{l}0.82(0.49-1.37) \\
0.45\end{array}$ & Reference & $\begin{array}{l}1.00(0.56-1.77) \\
1.00\end{array}$ & $\begin{array}{c}1.12(0.34-3.68) \\
0.85\end{array}$ \\
\hline $\begin{array}{l}\text { C69-C72: Malignant neoplasms of the eyes, brain, } \\
\text { and other parts of central nervous system }(n=1)\end{array}$ & - & - & Reference & - & - \\
\hline $\begin{array}{l}\text { C73-C75: Malignant neoplasms of the thyroid } \\
\text { and other endocrine glands }(n=1)\end{array}$ & - & - & Reference & - & - \\
\hline $\begin{array}{l}\text { C76-C80: Malignant neoplasms of ill-defined, } \\
\text { other secondary, and unspecified sites }(n=13)\end{array}$ & $\begin{array}{l}0.46(0.05-3.83) \\
0.47\end{array}$ & $\begin{array}{l}0.15(0.02-1.24) \\
0.08\end{array}$ & Reference & $\begin{array}{l}1.05(0.30-3.74) \\
0.93\end{array}$ & - \\
\hline $\begin{array}{l}\text { C81-C96: Malignant neoplasms of lymphoid, } \\
\text { hematopoietic, and related tissues }(n=15)\end{array}$ & $\begin{array}{l}2.43(0.48-12.3) \\
0.28\end{array}$ & $\begin{array}{c}1.91(0.47-7.71) \\
0.36\end{array}$ & Reference & $\begin{array}{c}1.59(0.26-9.63) \\
0.61\end{array}$ & $\begin{array}{c}7.22(0.72-72.4) \\
0.09\end{array}$ \\
\hline D00-D09: In situ neoplasms $(n=2)$ & - & - & Reference & - & - \\
\hline Unknown $(\mathrm{n}=71)$ & $\begin{array}{c}0.79(0.38-1.63) \\
0.53\end{array}$ & $\begin{array}{c}0.70(0.40-1.22) \\
0.21\end{array}$ & Reference & $\begin{array}{c}0.50(0.22-1.13) \\
0.09\end{array}$ & $\begin{array}{c}0.52(0.07-3.83) \\
0.52\end{array}$ \\
\hline
\end{tabular}

Models were adjusted for participants' age and gender, social history (smoking, alcohol consumption, and exercise), body mass index, comorbidities (hypertension, diabetes, and dyslipidemia) and family history of any cancer. Number in bold represents $\mathrm{p}<0.05$.

Table 5. Adjusted hazard ratios of developing any type and each type of cancer by sleep duration category stratified by age group from time-varying Cox proportional hazard model adjusting for different covariates.

\begin{tabular}{|c|c|c|c|c|c|}
\hline & \multicolumn{5}{|c|}{$\begin{array}{c}\text { Sleep duration category } \\
\text { Adjusted hazard ratio (95\% confidence interval) } \\
\text { p-value }\end{array}$} \\
\hline & $\leq 5$ hours & 5-6 hours & 6-7 hours & 7-8 hours & $\geq 8$ hours \\
\hline \multicolumn{6}{|l|}{ Younger $(50-64$ years $)(n=19,662)$} \\
\hline Any type of cancer $(\mathrm{n}=1,635)$ & $\begin{array}{l}0.94(0.82-1.09) \\
0.44\end{array}$ & $\begin{array}{l}0.87(0.78-0.98) \\
0.02\end{array}$ & Reference & $\begin{array}{l}1.10(0.94-1.29) \\
0.24\end{array}$ & $\begin{array}{c}1.04(0.67-1.59) \\
0.87\end{array}$ \\
\hline $\begin{array}{l}\text { C00-C14: Malignant neoplasms of the lips, } \\
\text { oral cavity and pharynx }(n=7)\end{array}$ & $\begin{array}{l}1.30(0.23-7.43) \\
0.77\end{array}$ & $\begin{array}{l}0.23(0.03-2.11) \\
0.20\end{array}$ & Reference & - & - \\
\hline $\begin{array}{l}\text { C15-C26: Malignant neoplasms of digestive } \\
\text { organs }(n=574)\end{array}$ & $\begin{array}{l}0.93(0.72-1.20) \\
0.58\end{array}$ & $\begin{array}{l}0.95(0.72-1.16) \\
0.64\end{array}$ & Reference & $\begin{array}{l}1.13(0.86-1.49) \\
0.37\end{array}$ & $\begin{array}{c}1.18(0.6--2.31) \\
0.64\end{array}$ \\
\hline $\begin{array}{l}\text { C30-C39: Malignant neoplasms of respiratory } \\
\text { and intrathoracic organs }(n=214)\end{array}$ & $\begin{array}{l}0.69(0.45-1.07) \\
0.10\end{array}$ & $\begin{array}{l}0.76(0.55-1.05) \\
0.09\end{array}$ & Reference & $\begin{array}{l}1.29(0.85-1.94) \\
0.23\end{array}$ & $\begin{array}{c}1.67(0.66-4.18) \\
0.28\end{array}$ \\
\hline $\begin{array}{l}\text { C40-C41: Malignant neoplasms of bone and } \\
\text { articular cartilage }(n=0)\end{array}$ & - & - & Reference & - & - \\
\hline $\begin{array}{l}\text { C43-C44: Melanoma and other malignant } \\
\text { neoplasms of the skin }(n=27)\end{array}$ & $\begin{array}{l}0.37(0.08-1.67) \\
0.20\end{array}$ & $\begin{array}{l}0.79(0.33-1.87) \\
0.59\end{array}$ & Reference & $\begin{array}{l}1.04(0.33-3.29) \\
0.94\end{array}$ & - \\
\hline $\begin{array}{l}\text { C45-C49: Malignant neoplasms of mesothe- } \\
\text { lial and soft tissue }(n=4)\end{array}$ & - & - & Reference & - & - \\
\hline $\begin{array}{l}\text { C50-C50: Malignant neoplasms of breast } \\
\text { tissue }(n=223)\end{array}$ & $\begin{array}{l}1.23(0.85-1.78) \\
0.27\end{array}$ & $\begin{array}{l}0.96(0.70-1.32) \\
0.80\end{array}$ & Reference & $\begin{array}{l}0.69(0.40-1.20) \\
0.19\end{array}$ & $\begin{array}{c}0.46(0.06-3.34) \\
0.44\end{array}$ \\
\hline $\begin{array}{l}\text { C51-C58: Malignant neoplasms of female } \\
\text { genital organs }(n=64)\end{array}$ & $\begin{array}{l}1.30(0.67-2.54) \\
0.44\end{array}$ & $\begin{array}{l}0.84(0.46-1.53) \\
0.56\end{array}$ & Reference & $\begin{array}{l}0.75(0.28-1.98) \\
0.56\end{array}$ & $\begin{array}{c}1.72(0.23-12.9) \\
0.60\end{array}$ \\
\hline $\begin{array}{l}\text { C60-C63: Malignant neoplasms of male geni- } \\
\text { tal organs }(n=331)\end{array}$ & $\begin{array}{l}0.97(0.71-1.34) \\
0.86\end{array}$ & $\begin{array}{l}0.74(0.56-0.97) \\
0.03\end{array}$ & Reference & $\begin{array}{l}1.30(0.94-1.81) \\
0.11\end{array}$ & $\begin{array}{c}0.78(0.29-2.14) \\
0.63\end{array}$ \\
\hline $\begin{array}{l}\text { C64-C68: Malignant neoplasms of the urinary } \\
\text { tract }(n=60)\end{array}$ & $\begin{array}{l}1.17(0.57-2.41) \\
0.67\end{array}$ & $\begin{array}{l}0.77(0.41-1.44) \\
0.41\end{array}$ & Reference & $\begin{array}{l}0.99(0.42-2.36) \\
0.99\end{array}$ & $\begin{array}{c}1.27(0.17-9.67) \\
0.82\end{array}$ \\
\hline $\begin{array}{l}\text { C69-C72: Malignant neoplasms of the eyes, } \\
\text { brain, and other parts of central nervous } \\
\text { system }(n=5)\end{array}$ & $\begin{array}{l}0.75(0.06-9.95) \\
0.83\end{array}$ & $\begin{array}{l}0.54(0.06-4.53) \\
0.57\end{array}$ & Reference & - & - \\
\hline $\begin{array}{l}\text { C73-C75: Malignant neoplasms of the thyroid } \\
\text { and other endocrine glands }(n=11)\end{array}$ & $\begin{array}{l}0.32(0.04-2.78) \\
0.30\end{array}$ & $\begin{array}{l}0.80(0.23-2.83) \\
0.74\end{array}$ & Reference & - & - \\
\hline $\begin{array}{l}\text { C76-C80: Malignant neoplasms of ill-defined, } \\
\text { other secondary, and unspecified sites }(n=11)\end{array}$ & $\begin{array}{l}0.65(0.13-3.32) \\
0.61\end{array}$ & $\begin{array}{l}0.28(0.06-1.39) \\
0.12\end{array}$ & Reference & $\begin{array}{l}0.45(0.05-3.81) \\
0.47\end{array}$ & - \\
\hline $\begin{array}{l}\text { C81-C96: Malignant neoplasms of lymphoid, } \\
\text { hematopoietic, and related tissues }(n=23)\end{array}$ & $\begin{array}{l}1.47(0.33-6.58) \\
0.62\end{array}$ & $\begin{array}{l}2.68(0.87-8.23) \\
0.09\end{array}$ & Reference & $\begin{array}{l}1.57(0.29-8.60) \\
0.60\end{array}$ & $\begin{array}{c}10.1(1.12-91.5) \\
0.04\end{array}$ \\
\hline
\end{tabular}


Table 5. Continued ...

\begin{tabular}{|c|c|c|c|c|c|}
\hline & \multicolumn{5}{|c|}{$\begin{array}{c}\text { Sleep duration category } \\
\text { Adjusted hazard ratio (95\% confidence interval) } \\
\text { p-value }\end{array}$} \\
\hline & $\leq 5$ hours & 5-6 hours & 6-7 hours & 7-8 hours & $\geq 8$ hours \\
\hline D00-D09: In situ neoplasms $(\mathrm{n}=2)$ & - & - & Reference & - & - \\
\hline Unknown $(\mathrm{n}=81)$ & $\begin{array}{l}0.75(0.37-1.52) \\
0.42\end{array}$ & $\begin{array}{l}1.01(0.60-1.69) \\
0.97\end{array}$ & Reference & $\begin{array}{l}1.32(0.65-2.67) \\
0.44\end{array}$ & - \\
\hline \multicolumn{6}{|l|}{ Elderly ( 65 years or older $)(n=5,211)$} \\
\hline Any type of cancer $(n=909)$ & $\begin{array}{l}0.95(0.76-1.19) \\
0.66\end{array}$ & $\begin{array}{l}1.12(0.95-1.31) \\
0.19\end{array}$ & Reference & $\begin{array}{l}1.04(0.86-1.24) \\
0.71\end{array}$ & $\begin{array}{l}1.16(0.81-1.67) \\
0.42\end{array}$ \\
\hline $\begin{array}{l}\text { C00-C14: Malignant neoplasms of the lips, } \\
\text { oral cavity and pharynx }(n=6)\end{array}$ & - & $\begin{array}{l}0.40(0.04-4.07) \\
0.44\end{array}$ & Reference & $\begin{array}{l}0.43(0.04-4.40) \\
0.48\end{array}$ & - \\
\hline $\begin{array}{l}\text { C15-C26: Malignant neoplasms of digestive } \\
\text { organs }(n=326)\end{array}$ & $\begin{array}{l}0.92(0.62-1.35) \\
0.66\end{array}$ & $\begin{array}{c}1.33(1.02-1.75) \\
0.04\end{array}$ & Reference & $\begin{array}{l}1.03(0.75-1.41) \\
0.87\end{array}$ & $\begin{array}{c}1.74(1.03-2.96) \\
0.04\end{array}$ \\
\hline $\begin{array}{l}\text { C30-C39: Malignant neoplasms of respiratory } \\
\text { and intrathoracic organs }(n=110)\end{array}$ & $\begin{array}{l}1.14(0.60-2.16) \\
0.69\end{array}$ & $\begin{array}{l}1.47(0.93-2.31) \\
0.10\end{array}$ & Reference & $\begin{array}{l}0.77(0.43-1.39) \\
0.38\end{array}$ & $\begin{array}{c}0.83(0.25-2.69) \\
0.75\end{array}$ \\
\hline $\begin{array}{l}\text { C40-C41: Malignant neoplasms of bone and } \\
\text { articular cartilage }(n=1)\end{array}$ & - & - & Reference & - & - \\
\hline $\begin{array}{l}\text { C43-C44: Melanoma and other malignant } \\
\text { neoplasms of the skin }(n=21)\end{array}$ & $\begin{array}{c}1.47(0.44-4.86) \\
0.53\end{array}$ & $\begin{array}{l}0.78(0.26-2.37) \\
0.67\end{array}$ & Reference & $\begin{array}{c}0.63(0.17-2.34) \\
0.49\end{array}$ & - \\
\hline $\begin{array}{l}\text { C45-C49: Malignant neoplasms of mesothe- } \\
\text { lial and soft tissue }(n=1)\end{array}$ & - & - & Reference & - & - \\
\hline $\begin{array}{l}\text { C50-C50: Malignant neoplasms of breast } \\
\text { tissue }(n=56)\end{array}$ & $\begin{array}{l}0.82(0.35-1.95) \\
0.66\end{array}$ & $\begin{array}{l}1.06(0.57-1.98) \\
0.85\end{array}$ & Reference & $\begin{array}{l}0.81(0.35-1.84) \\
0.61\end{array}$ & $\begin{array}{c}0.78(0.10-5.82) \\
0.81\end{array}$ \\
\hline $\begin{array}{l}\text { C51-C58: Malignant neoplasms of female } \\
\text { genital organs }(n=17)\end{array}$ & $\begin{array}{c}1.37(0.21-8.76) \\
0.74\end{array}$ & $\begin{array}{l}3.35(0.87-12.8) \\
0.08\end{array}$ & Reference & $\begin{array}{c}3.07(0.67-14.0) \\
0.15\end{array}$ & - \\
\hline $\begin{array}{l}\text { C60-C63: Malignant neoplasms of male geni- } \\
\text { tal organs }(n=242)\end{array}$ & $\begin{array}{l}0.85(0.54-1.32) \\
0.46\end{array}$ & $\begin{array}{c}0.68(0.49-0.96) \\
0.03\end{array}$ & Reference & $\begin{array}{l}1.15(0.83-1.59) \\
0.39\end{array}$ & $\begin{array}{c}0.82(0.38-1.76) \\
0.60\end{array}$ \\
\hline $\begin{array}{l}\text { C64-C68: Malignant neoplasms of the urinary } \\
\text { tract }(n=69)\end{array}$ & $\begin{array}{c}1.02(0.43-2.43) \\
0.96\end{array}$ & $\begin{array}{l}1.38(0.75-2.54) \\
0.30\end{array}$ & Reference & $\begin{array}{l}1.48(0.77-2.84) \\
0.24\end{array}$ & $\begin{array}{c}1.67(0.49-5.67) \\
0.41\end{array}$ \\
\hline $\begin{array}{l}\text { C69-C72: Malignant neoplasms of the eyes, } \\
\text { brain, and other parts of central nervous } \\
\text { system }(n=1)\end{array}$ & - & - & Reference & - & - \\
\hline $\begin{array}{l}\text { C73-C75: Malignant neoplasms of the thyroid } \\
\text { and other endocrine glands }(n=7)\end{array}$ & $\begin{array}{l}2.65(0.35-20.3) \\
0.35\end{array}$ & $\begin{array}{l}2.08(0.32-13.4) \\
0.44\end{array}$ & Reference & - & - \\
\hline $\begin{array}{l}\text { C76-C80: Malignant neoplasms of ill-defined, } \\
\text { other secondary, and unspecified sites }(n=10)\end{array}$ & $\begin{array}{l}0.80(0.08-7.55) \\
0.85\end{array}$ & $\begin{array}{l}0.33(0.04-3.11) \\
0.34\end{array}$ & Reference & $\begin{array}{l}1.67(0.39-7.25) \\
0.49\end{array}$ & - \\
\hline $\begin{array}{l}\text { C81-C96: Malignant neoplasms of lymphoid, } \\
\text { hematopoietic, and related tissues }(n=6)\end{array}$ & $\begin{array}{l}1.19(0.11-13.4) \\
0.89\end{array}$ & - & Reference & $\begin{array}{l}1.95(0.27-14.0) \\
0.51\end{array}$ & $\begin{array}{c}7.33(0.65-83.1) \\
0.11\end{array}$ \\
\hline D00-D09: In situ neoplasms $(\mathrm{n}=0)$ & - & - & Reference & - & - \\
\hline Unknown $(\mathrm{n}=36)$ & $\begin{array}{l}1.14(0.40-3.22) \\
0.81\end{array}$ & $\begin{array}{l}1.30(0.61-2.77) \\
0.50\end{array}$ & Reference & $\begin{array}{l}0.44(0.12-1.54) \\
0.20\end{array}$ & $\begin{array}{l}0.88(0.11-6.75) \\
0.90\end{array}$ \\
\hline
\end{tabular}

Models were adjusted for participants' age and gender, social history (smoking, alcohol consumption, and exercise), body mass index, comorbidities (hypertension, diabetes, and dyslipidemia) and family history of any cancer. Number in bold represents $\mathrm{p}<0.05$.

with malignant neoplasms of lymphoid, hematopoietic, and related tissues among females in our study population, which was supported by a previous study [13]. Although this association was not observed statistically in males, a relatively high HR and low p-value suggested that the association may exist if we have enough incidences of cancer. The potential mechanism of an increased risk for malignant neoplasms of lymphoid, hematopoietic, and related tissues may be as disturbance of immune-inflammation valance due to long sleep duration $[14,15]$. Finally, short sleep duration was associated with a lower incidence of malignant neoplasms of male genital organs, mainly prostate cancer. Two cohort studies examined the association between short sleep duration and prostate cancer but failed [16, 17]. However, short sleepers had lower HRs of prostate cancer compared to normal sleep duration in both studies, although these were not statistically significant. Although there could be certain associations between sleep duration and each type of cancer as we discussed above, potential mechanisms are still obscure.

Our study has some limitations. First, the information about sleep duration was self-reported and not objective. Although almost all previous studies also used self-reported sleep durations, measured sleep durations may be different, causing non-differential bias. Second, our data did not 
include variables that may affect sleep duration, such as a history of depression. However, because depression itself would not be associated with or cause cancer, this would not be a confounding factor for which we should have adjusted the model. In addition, we would have a multiple-testing problem. Although our primary outcome was any type of cancer development, we had 16 secondary outcomes. Such a large number of outcomes may increase the chance of false positives. Furthermore, this study was exploratory; therefore, we cannot mention causality about the association. Moreover, although we adjusted for potential covariates in the multivariable analyses, the information on the dietary habit or composition which may be another important factor that would affect cancer development through the influence on the intestinal microbiome community was lacking. This covariate may differentiate the findings when it is in the model. Finally, our population was mostly Japanese, thus, we cannot generalize our results to the Western population. However, we can add some evidence on the research about the association between sleep duration and development of cancer in the Asian population.

In conclusion, according to our longitudinal study, there is no clear association between sleep duration and subsequent development of any type of cancer. However, sleep duration was associated with certain types of cancer, such as that of digestive organs, lymphoid, hematopoietic, and related tissues in females; and genital organs in males.

\section{References}

[1] CHEN Y, TAN F, WEI L, LI X, LYU Z et al. Sleep duration and the risk of cancer: a systematic review and meta-analysis including dose-response relationship. BMC Cancer 2018; 18: 1149. https://doi.org/10.1186/s12885-018-5025-y

[2] LU C, SUN H, HUANG J, YIN S, HOU W et al. Long-Term Sleep Duration as a Risk Factor for Breast Cancer: Evidence from a Systematic Review and Dose-Response MetaAnalysis. Biomed Res Int 2017; 2017: 4845059. https://doi. org/10.1155/2017/4845059

[3] ZHAO H, YIN JY, YANG WS, QIN Q, LI TT et al. Sleep duration and cancer risk: a systematic review and meta-analysis of prospective studies. Asian Pac J Cancer Prev 2013; 14: 7509-7515. https://doi.org/10.7314/apjcp.2013.14.12.7509

[4] LUOJUS MK, LEHTO SM, TOLMUNEN T, ERKKILA AT, KAUHANEN J. Sleep duration and incidence of lung cancer in ageing men. BMC Public Health 2014; 14: 295. https://doi. org/10.1186/1471-2458-14-295

[5] SHEN J, CHRISMAN M, WU X, CHOW WH, ZHAO H. Sleep duration and risk of cancer in the Mexican American Mano-a-Mano Cohort. Sleep Health 2019; 5: 78-83. https:// doi.org/10.1016/j.sleh.2018.09.004
[6] ZHANG X, GIOVANNUCCI EL, WU K, GAO X, HU F et al. Associations of self-reported sleep duration and snoring with colorectal cancer risk in men and women. Sleep 2013; 36: 681-688. https://doi.org/10.5665/sleep.2626

[7] WANG P, REN FM, LIN Y, SU FX, JIA WH et al. Night-shift work, sleep duration, daytime napping, and breast cancer risk. Sleep Med 2015; 16: 462-468. https://doi.org/10.1016/j. sleep.2014.11.017

[8] Kobayashi D, Takahashi O, Shimbo T, Okubo T, Arioka H et al. High sleep duration variability is an independent risk factor for weight gain. Sleep Breath 2013; 17: 167-172. https:// doi.org/10.1007/s11325-012-0665-7

[9] KRAUSE N, INGERSOLL-DAYTON B, LIANG J, SUGISAWA H. Religion, social support, and health among the Japanese elderly. J Health Soc Behav 1999; 40: 405-421.

[10] VON RUESTEN A, WEIKERT C, FIETZE I, BOEING H. Association of sleep duration with chronic diseases in the European Prospective Investigation into Cancer and $\mathrm{Nu}-$ trition (EPIC)-Potsdam study. PLoS One 2012; 7: e30972. https://doi.org/10.1371/journal.pone.0030972

[11] WHO EXPERT CONSULTATION. Appropriate body-mass index for Asian populations and its implications for policy and intervention strategies. Lancet 2004; 363: 157-163. https://doi.org/10.1016/S0140-6736(03)15268-3

[12] MA QQ, YAO Q, LIN L, CHEN GC, YU JB. Sleep duration and total cancer mortality: a meta-analysis of prospective studies. Sleep Med 2016; 27-28: 39-44. https://doi. org/10.1016/j.sleep.2016.06.036

[13] GU F, XIAO Q, CHU LW, YU K, MATTHEWS CE et al. Sleep Duration and Cancer in the NIH-AARP Diet and Health Study Cohort. PLoS One 2016; 11: e0161561. https:// doi.org/10.1371/journal.pone.0161561

[14] MCNEIL J, BARBERIO AM, FRIEDENREICH CM, BRENNER DR. Sleep and cancer incidence in Alberta's Tomorrow Project cohort. Sleep 2019; 42: zsy252. https://doi. org/10.1093/sleep/zsy252

[15] LENG Y, AHMADI-ABHARI S, WAINWRIGHT NW, CAPPUCCIO FP, SURTEES PG et al. Daytime napping, sleep duration and serum $\mathrm{C}$ reactive protein: a populationbased cohort study. BMJ Open 2014; 4: e006071. https://doi. org/10.1136/bmjopen-2014-006071

[16] MARKT SC, FLYNN-EVANS EE, VALDIMARSDOTTIR UA, SIGURDARDOTTIR LG, TAMIMI RM et al. Sleep Duration and Disruption and Prostate Cancer Risk: a 23Year Prospective Study. Cancer Epidemiol Biomarkers Prev 2016; 25: 302-308. https://doi.org/10.1158/1055-9965.EPI14-1274

[17] MARKT SC, GROTTA A, NYREN O, ADAMI HO, MUCCI LA et al. Insufficient Sleep and Risk of Prostate Cancer in a Large Swedish Cohort. Sleep 2015; 38: 1405-1410. https:// doi.org/10.5665/sleep.4978 